\title{
Cultura Ciudadana: Arquitectura, Ciudad y Niños. Experiencias en San José, Costa Rica
}

\author{
Citizens Culture: Architecture, City and Children. Experiences \\ in San Jose, Costa Rica
}

\section{Cultura da Cidadão: Arquitetura, Cidade e Crianças. Experiências em San Jose, Costa Rica}

Ximena C. Pizarro

Colegio de Arquitectos de Costa Rica

\begin{abstract}
Tener ciudades humanizadas con calidad de vida para todos depende, definitivamente de proponer un cambio en los niños y jóvenes desde el inicio a través de los procesos educativos, utilizando nuevas formas de aprender de una manera lúdica y participativa. Este artículo presenta talleres realizados en San José, Costa Rica: en escuela pública, escuela privada y espacio público. Actividades que permiten identificar los aportes de esta población en las soluciones sostenibles de las ciudades con base en lo compartido y aprendido en los talleres. Se concluye que los conocimientos adquiridos se asimilaron, generando opinión, sentido crítico y creativo al respecto.
\end{abstract}

Descriptores: Comunidad, Niño, Cultura de paz, Desarrollo participativo, Población urbana.

To have humanized cities with quality of life for all depends on the change of children and young people through educational processes, using new ways to learn in a playful and participative way. This article presents workshops held in San José, Costa Rica: in public school, private school and public space. Activities that allow the identification of the contributions of this population in the sustainable solutions of the cities based on the shared and learned in the workshops. In conclusion, the acquired knowledge was assimilated and it generated opinion, critical and creative sense in this respect.

Keywords: Community, Child, Peace culture, Participatory development, Urban population.

Ter cidades humanizados com qualidade de vida para todos passa pela proposta de uma alteração, desde cedo, dos processos educacionais nas crianças e nos jovens, utilizando novas formas de aprendizagem divertidas e participativas. Este artigo apresenta workshops realizados em San Jose, Costa Rica: nas escolas públicas, escolas particulares e em espaço público. Atividades que permitem identificar as contribuições da população das cidades com base na partilha e a aprendizagem nas oficinas. Conclui-se que o conhecimento adquirido foi assimilado, criando opiniões e pensamento crítico e criativo nesse âmbito.

Palavras-chave: Comunidade, Criança, Cultura de paz, Desenvolvimento participativo, População urbana.

*Contacto: xcpizarro@gmail.com

ISSN: 2254-3139

www.rinace.net/riejs/

revistas.uam.es/riejs
Recibido: $\quad 7$ de enero 2017

$1^{\text {a }}$ Evaluación: 12 de febrero 2017

$2^{\text {a }}$ Evaluación 25 de febrero 2017

Aceptado: $\quad 28$ de febrero 2017 


\section{Introducción}

Este trabajo se define dentro de las áreas del saber relacionadas con la Arquitectura y el Urbanismo, específicamente orientado hacia los procesos educativos dirigidos para la niñez y la juventud en temas de ciudad y sus sistemas, integrando conceptos, lenguajes, técnicas y otros componentes físicos y sociales del entorno construido del país para incentivar la comprensión, el conocimiento, la valoración, el sentido crítico y el cuido del mismo.

Las diversas actividades que se realizan pretenden aportar conocimientos a la niñez y a la juventud en relación con el espacio construido, la infraestructura, las ciudades, la sostenibilidad, la vida en comunidad, el patrimonio y de esta manera motivar acciones desde tempranas edades aplicadas en su entorno inmediato como lo son las comunidades, escuelas y colegios. De esta manera anticipar resultados a largo alcance en la calidad de vida de las personas. Para realizar esta práctica de la sensibilización se motiva, se escucha, se intercambian ideas, se explora, se experimenta, se comparte, se despierta el asombro, el descubrir, desarrollándolo a través de actividades participativas como el juego, el teatro, la música, los talleres, las dinámicas, las charlas, el material didáctico, entre otros, todos ellos medios atractivos y de mucho interés para los niños, las niñas y los jóvenes. Los participantes motivados aprenden e intercambian ideas, de esta manera se fortalecen los valores y conceptos como la sostenibilidad ambiental y cultural obteniendo así niños y jóvenes preparados, con conocimiento hacia el análisis del espacio construido y su entorno urbano. Se obtiene de esta manera una cultura ciudadana.

La justificación de esta propuesta se basa en la importancia de implementar una nueva forma de aprender a través de la vivencia, de la experiencia, de hacer utilizando los sentidos, generando una actitud crítica, de opinión y sensible en los usuarios de las ciudades desde niños, desarrollando en ellos el interés en practicar diariamente acciones que benefician al ambiente y a la comunidad en general.

\section{Objetivo general}

Inculcar conocimientos de la arquitectura y el urbanismo en diferentes escuelas, colegios y comunidades y espacios públicos de la ciudad de San José, Costa Rica dirigido a la niñez y a la juventud, con base en el entorno construido nacional, destacando en lo arquitectónico, la infraestructura, la ciudad y la vida en comunidad.

\section{Objetivos específicos}

- Compartir el conocimiento de la arquitectura y el urbanismo con los ciudadanos desde tempranas edades para la vida en comunidad, lo que se denomina cultura ciudadana, cultura urbana, construir ciudadanía.

- Sensibilizar a los niños, niñas y jóvenes para una adecuada interpretación de los conceptos de arquitectura, infraestructura, ciudad, ambiente y sostenibilidad.

- Generar un sentido crítico en el niño, la niña y los jóvenes a la hora de utilizar y ser usuario permanente del espacio público, de su casa, de su barrio, de su ciudad por medio del juego y la expresión artística. 
- Obtener propuestas e ideas urbanas de parte de los niños, niñas y jóvenes de las escuelas, de las comunidades, para mejorar el barrio, el entorno inmediato y la ciudad.

- Lograr integración y participación del niño y el joven como personas con derechos de opinión hacia su comunidad.

- Comprender la importancia de realizar trabajos en conjunto, aplicando sus ideas en propuestas concretas y así ser parte activa de los cambios del barrio, de la comunidad.

\section{Materiales y métodos}

Los talleres se realizaron con materiales reciclados y otros muy sencillos: cajas y láminas de cartón, papeles de colores, piezas de madera, papel periódico, vasos y platos plásticos, tapas plásticas, etc. Además de materiales facilitados como goma, masking tape, telas, títeres, tijeras, lápices de colores, pinturas, entre otros y el material más importante utilizado fueron las ideas, la expresión corporal, el juego, la disposición a divertirse, participar y aprender juntos.

Los métodos participativos y de creatividad utilizados en cada sitio permitieron la experimentación y resaltaron los sentidos y las vivencias en los participantes, actividades que generalmente no se ponen en práctica, pues se mantiene fuertemente en la educación actual de nuestros países, el sistema tradicional donde el maestro es quien dirige y manda en la clase, al menos en nuestra región centroamericana es el común denominador; aunque poco a poco se comienzan a ver propuestas diferentes donde el niño pasa a ser parte activa del proceso de aprendizaje. Se comienza a ver cambio en el proceso educativo, de una manera activa, es decir donde la retroalimentación es mutua siguiendo un diálogo e intercambio de experiencias del tipo profesor- estudiante y viceversa, de igual a igual, donde el tema aprender se convierte en algo que entusiasma, que asombra, que interesa y donde el espacio que habitamos se comienza a entender como algo fundamental que se usa diariamente y que en muchas ocasiones pasa desapercibido, ese espacio denominado, casa, barrio, escuela, comunidad, país. De acuerdo con Räsänen (2014) "Se necesitan sentidos para percibir el espacio. Las primeras experiencias que las personas tienen de espacio es el vientre de la madre” (p. 33).

El procedimiento realizado en las tres locaciones que se presentan se enumera de la siguiente manera:

- Reunirse para conversar el tema ciudad e intercambio de ideas (retroalimentación), espacio en el cual de una manera espontánea se conversa el tema y se opina libremente al respecto.

- Presentación del tema (conceptualización) el cual en algunos casos se apoya con presentaciones de obras de teatro y títeres o cualquier otra forma de manifestación artística,

- Luego la puesta en práctica del tema, se construye la idea. Se materializa de diversas formas: modelos, dibujos, poesías, historias, realización de dinámicas lúdicas del tema y otros. 
- Se realiza discusión y análisis de lo realizado y experimentado, se propone la manera de poner en práctica de los conceptos o temas estudiados y se realiza valoración de los mismos.

La población costarricense como usuaria de los espacios (públicos y privados), y de los elementos arquitectónicos que los conforman, demuestra su falta de conocimiento y sensibilidad ante el mismo, lo que se traduce en una falta de cultura que se manifiesta como síntomas de una insensibilidad ante el espacio y la comunidad, tema que se refleja de igual manera en muchos de los países de América. El deterioro del ambiente ha provocado desgaste en nuestras ciudades y descuido por parte de los usuarios lo que indica un desapego y falta de apropiación de las mismas. Esto se puede ver reflejado en la inseguridad por ejemplo, en las nuevas formas de vida en comunidad, en las propuestas de barrios cerrados por muros perimetrales que no permiten la comunicación, la integración, barrios que niegan al otro, que limita la interrelación a través de un gran muro, donde poco a poco se van perdiendo los espacios de encuentro, los espacios de juegos, tema que los niños perciben muy bien y por los cuales se debe trabajar fuertemente desde nuestra profesión, por una ciudad inclusiva y participativa, por una ciudad igualitaria y para todos, donde todos somos parte de ella. Ciudades que reflejen nuestra identidad, nuestra cultura.

Si se aplica el potencial de los procesos educativos para tocar estos temas y se valora más la importancia del trabajo de las escuelas en cada comunidad, se debe considerar, al igual que otras organizaciones nacionales e internacionales, que la oportunidad de lograr un cambio permanente en nuestras ciudades, es precisamente, mediante un proceso formativo dirigido a la niñez y la juventud, a través de diversos proyectos aplicados desde la escuela, la comunidad y los municipios. Los niños y los jóvenes deben hacer respetar sus derechos fundamentales como lo son el derecho a expresarse, a opinar, a una cultura, a la educación, a jugar, a ser parte activa en las decisiones de la ciudad, lo cual se puede hacer en una forma lúdica, dinámica e interactiva como puede ser la utilización de diferentes expresiones artísticas para tal fin, así lo menciona Subirats, socióloga, gestora pública y política catalana cuando dice, en el proyecto Ciudades Educadoras: un proyecto cargado de futuro, lo siguiente: "Nos hallamos al inicio de una nueva manera de entender la educación y los recursos educativos, y que, como en tantas cosas, los ayuntamientos pueden, deben y ya están siendo pioneros en el desarrollo de las nuevas posibilidades" (Subirats, 2007, p. 59). Los municipios deben ser apoyo fundamental en esta tarea.

Para lograr un cambio de perspectiva, un cambio de paradigma, un cambio de lenguaje que nos haga reflexionar en actuar diferente, se plantea desarrollar acciones prácticas denominadas talleres lúdico creativos a realizar en escuelas, comunidades y/o espacios públicos, talleres que permitirán a los participantes en forma activa y directa se involucren en la creación de ambientes sostenibles con calidad arquitectónica, amigable para el ser humano y respetuosa de su entorno y sean conscientes del valor de su identidad, como lo define Martín-Baró (2004) en su libro "Acción e ideología: Psicología social desde Centroamérica" donde plantea que "la identidad es, por consiguiente, el enraizamiento de la persona en un determinado mundo de significaciones así como en una determinada red de relaciones sociales" (p. 121), socializaciones de cada vez más se realizan de manera virtual junto al acelerado paso de la vida diaria, lo que sumado al protagonismo del automóvil, le va restando poco a poco lo humano y cercano a la ciudad. De ahí estas propuestas que se realizaron con el fin de obtener los siguientes impactos: 
- Educativo: que los niños, las niñas y los jóvenes de la comunidad se fortalezcan en el área de aprendizaje con efectos directos en las acciones que los estudiantes tendrán en su entorno inmediato, su casa, su barrio, su comunidad, detectándolos, haciendo buen uso de ellos y protegiéndolos con un sentido de apropiación del mismo.

- Socio-Cultural: fortalecimiento de los conceptos y rescate de la identidad, respeto de lugar, valorización del hábitat construido, de las oportunidades vivenciales que lo rodean, ser difusores de este conocimiento hacia la comunidad en general y creer en el trabajo en equipo para soluciones de objetivos comunes. Humanizar la ciudad, hacerla más cercana y amigable.

- Ambiental: reforzar el área del conocimiento de la sostenibilidad dentro del medio vivido. Utilización de energías alternativas en las actividades del diario vivir.

Con el sentido de:

- Que los niños, las niñas y los jóvenes posean información necesaria para saber convivir en comunidad y solucionar los problemas en conjunto.

- Saber aplicar diferentes alternativas para ayudar el medio ambiente y al ahorro energético tales como: reciclaje de desechos, ahorro de energía, ahorro de agua, sistemas de transportes alternativos.

- Aplicar conocimientos adquiridos en proyectos específicos dentro de su escuela y de su comunidad y saber identificar elementos primordiales (pulmones verdes, espacios de encuentro, aceras, y otros) para el funcionamiento óptimo de un barrio, de una comunidad y saber cuidarlos, respetarlos y mantenerlos.

- Entender la importancia de trabajar en equipo para objetivos comunes que benefician a todos.

A continuación se describen los tres talleres:

Taller 1. Visita a 20 Escuelas de Atención Prioritaria (escuelas ubicadas en general en el sector sur de la ciudad, en barrios de pobreza extrema) localizados en la provincia de San José, Costa Rica donde se realizaron las siguientes actividades: Obra de teatro y títeres "Una comunidad para todos", montaje artístico que se realizó específicamente para este proyecto, Taller de diálogo con los niños (estas dos actividades se realizaron al total de la población de la Escuela), Taller lúdico creativo (se realizó con el grupo o sección de segundo ciclo seleccionado por la Escuela (35 estudiantes en promedio por grupo), y por último luego de un mes, se regresó al sitio por la propuesta de ciudad que cada Escuela planteó construida únicamente con material de reciclaje. Este proyecto tuvo una duración de 6 meses en el año 2008, fue patrocinado por PROARTES (Programa Nacional para el Desarrollo de las Artes), avalado por el Ministerio de Educación Pública (MEP) y por el Colegio de Arquitectos de Costa Rica, específicamente por la Comisión de Espacio Construido y Niñez. En esta propuesta se recopiló la siguiente información:

- Los niños, niñas y jóvenes se organizaron y reflexionaron acerca de los diferentes temas que querían presentar. 
- Los estudiantes utilizaron material reciclado que se les facilitó y agregaron otros que ellos mismos propusieron.

- Los estudiantes trabajaron con profesores de varias materias en conjunto como los siguientes: Matemáticas, Español, Estudios sociales y Artes, donde se evidenció que pocas veces se trabajan en equipo temas del currículo escolar formal. De igual manera los participantes indicaron el interés que tuvieron al darse cuenta que en la ciudad se pueden aplicar los diversos temas que aprenden en clase, donde analizaron temas como proporción, historia, rotulación, organización y color de la ciudad, entre otros.

- Los estudiantes plantearon un centro de ciudad donde enlistaban una serie de cambios y peticiones que proponen hacer a la ciudad. Aparece entonces el uso de alternativas de transporte como lo es el tren y la bicicleta.

En la figura 1, A) se presenta una escena de la obra de títeres "Una comunidad para todos" presentada en las escuelas; B) los estudiantes trabajando su propuesta de ciudad; C) y D) son dos ejemplos de propuestas de ciudad.

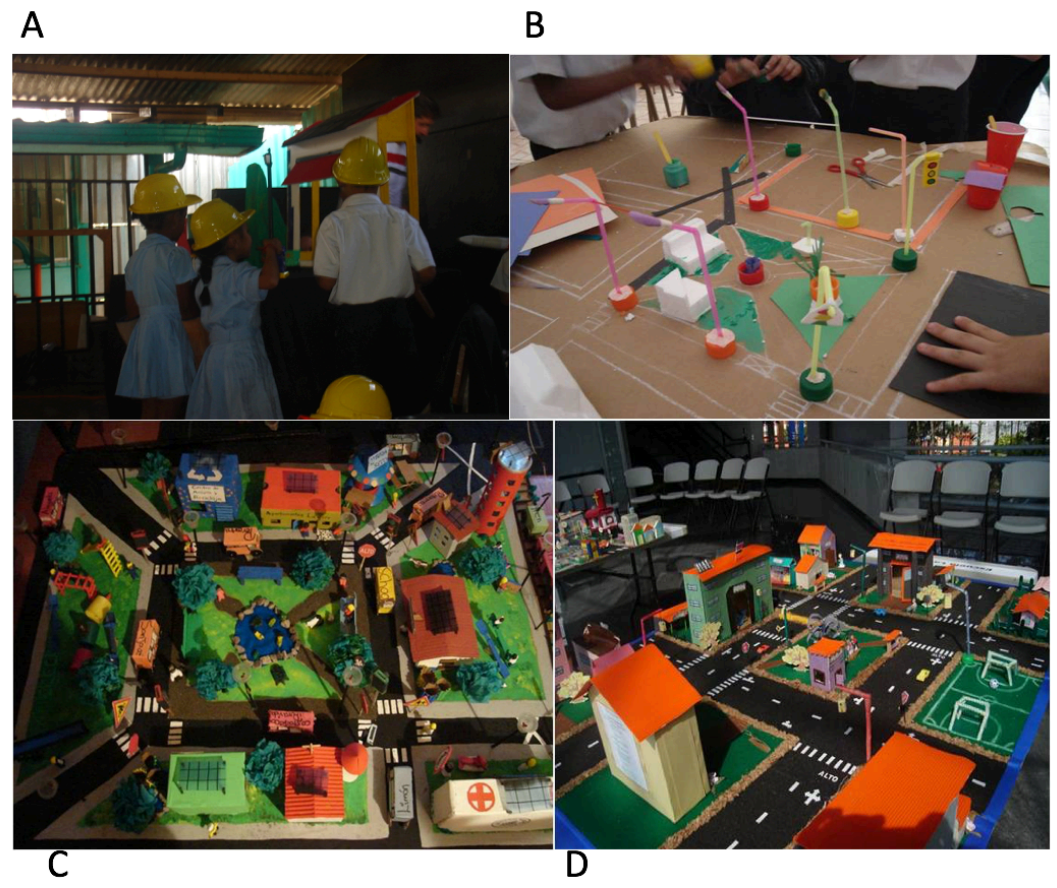

Figura 1. Taller en escuelas de atención prioritaria. Fuente: Elaboración propia.

Taller 2. Festival Internacional de las Artes de 2012, Taller "La ciudad a nuestra medida" donde los niños, niñas y jóvenes visitantes del Festival, realizado en la Parque Metropolitano La Sabana en San José Costa Rica, el espacio público y pulmón verde más grande del centro de San José, los niños visitantes construyeron una ciudad de $200 \mathrm{~m} 2$ (tarima de $20 \mathrm{~m} \times 10 \mathrm{~m}$ ) compartiendo sus ideas durante 10 días consecutivos con material reciclado. Los participantes de este taller representan a todos los niveles sociales de nuestra ciudad pues se trataba de una actividad abierta a todo público. En este taller se realizaron las siguientes actividades: Presentación de obra de teatro y títeres; Conversatorio con los niños acerca del tema arquitectura y ciudad; Taller de construcción de propuestas para la ciudad con material reciclado y colocación de la 
propuesta en la gran ciudad, y por último se debía dejar en forma escrita y/o en dibujo una idea para la ciudad en el juego "Bosque de ideas", con el fin de recaudar la información del público infantil visitante. Esta actividad se realizó con el apoyo del Festival quien facilitó el espacio físico, los materiales fueron donaciones realizadas y recolectadas entre el público en general y empresa privada que apoyó. Las actividades fueron realizadas junto a 20 estudiantes universitarios de diferentes carreras realizando su trabajo comunal requisito en las Universidades del país.

En la tabla 1 se observa la participación al taller "La ciudad a nuestra medida" fue de nueve escuelas diarias públicas y privadas con un promedio de 100 estudiantes por escuela para un total de 900 estudiantes diarios lo que da un total general de 5400 estudiantes en 6 días hábiles, y si se agrega el público itinerante familiar en fin de semana de 6000 personas en dos fines de semana (niños, adultos y adultos mayores), se obtiene un total de 11400 visitantes en 10 días, factor importante para el desarrollo de este proyecto aportando gran cantidad de ideas, y aumentando los objetivos propuestos.

Tabla 1. Asistencia al taller "La ciudad a nuestra medida"

\begin{tabular}{lrr}
\hline \multicolumn{1}{c}{ Días } & \multicolumn{2}{c}{ NúMERO DE PERSONAS } \\
\cline { 2 - 3 } & Por día & Total \\
\hline Lunes a Viernes (6 días hábiles) & 900 & 5,400 \\
Sábado a Domingo (4 días) & 1,500 & 6,000 \\
Total (10 días) & - & 11,400 \\
\hline Fur
\end{tabular}

Fuente: Elaboración propia.

En la figura 2, A) presenta una propuesta gráfica donde se solicitan más juegos para niños en la ciudad; B) idea de niño solicitando una ciudad feliz; C) Juego: el bosque de ideas, donde los participantes dejan propuesta en forma escrito y/o dibujo; D) vista de la ciudad construida en diez días por los niños visitantes, y E) niño propone una ciudad para caminar.

Taller 3. Arqui-ticos del IEM (Instituto Educativo Moderno), Taller realizado en escuela privada, dirigida a un público de clase media, localizada en el sector este de la capital, específicamente en Concepción de Tres Ríos, en la ciudad de San José de Costa Rica, conformado por 27 niños de segundo ciclo de primaria y con una duración de 4 meses cada curso, compuesto por una clase semanal de una hora. Curso que se realiza en forma permanente desde el año 2014. Este taller tiene como fin descubrir el sentido de la arquitectura como experiencia humana para la vida en comunidad, en la propuesta se realizaron las siguientes actividades: Conocer y recorrer la ciudad (mapa), Compartir con amigos el conocimiento de la ciudad (intercambio con niños de otras partes), Construcción con el cuerpo (analogías), Estudio formas geométricas (el triángulo), Construir con telas entre los árboles (aplicación del triángulo y del tema tensión aplicada y diseño de la estructura), Escultura con cajas (diseño), Dibujo y rediseño mi casa (función - forma), Cajas ciudad Mondrian (artista-arte), Los colores de la noche (observar-ciudad mochila), Ciudad Mondrian (planificación-arte-arquitectura-ciudad), todas actividades realizadas por medio de la experiencia, de lo lúdico y de la reflexión.

En la figura 3 aparece, A) los colores de la ciudad en la noche (ciudad mochila); B) cajas Mondrian (artista, arte); C) los estudiantes explicando su propuesta de ciudad; D) estudiando el triángulo (estructura, forma, volumen), y E) compartiendo con los amigos del exterior el conocimiento de la ciudad de San José. 


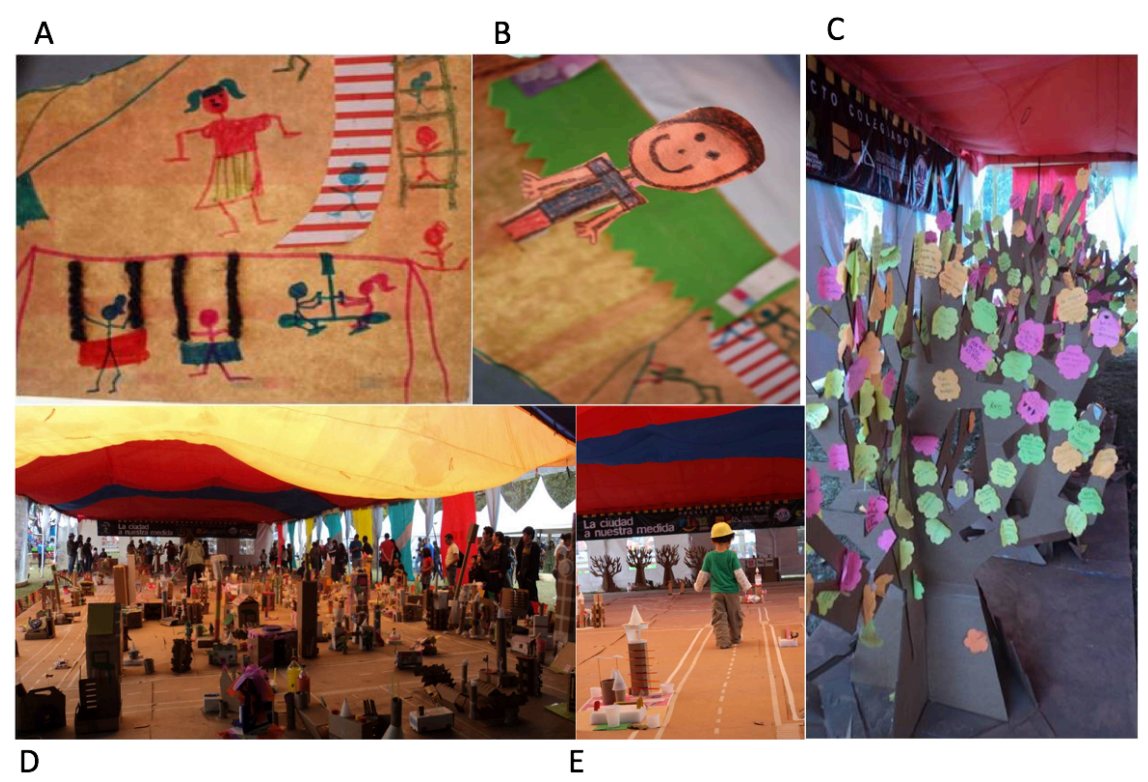

Figura 2. Taller La ciudad a nuestra medida Fuente: Elaboración propia.

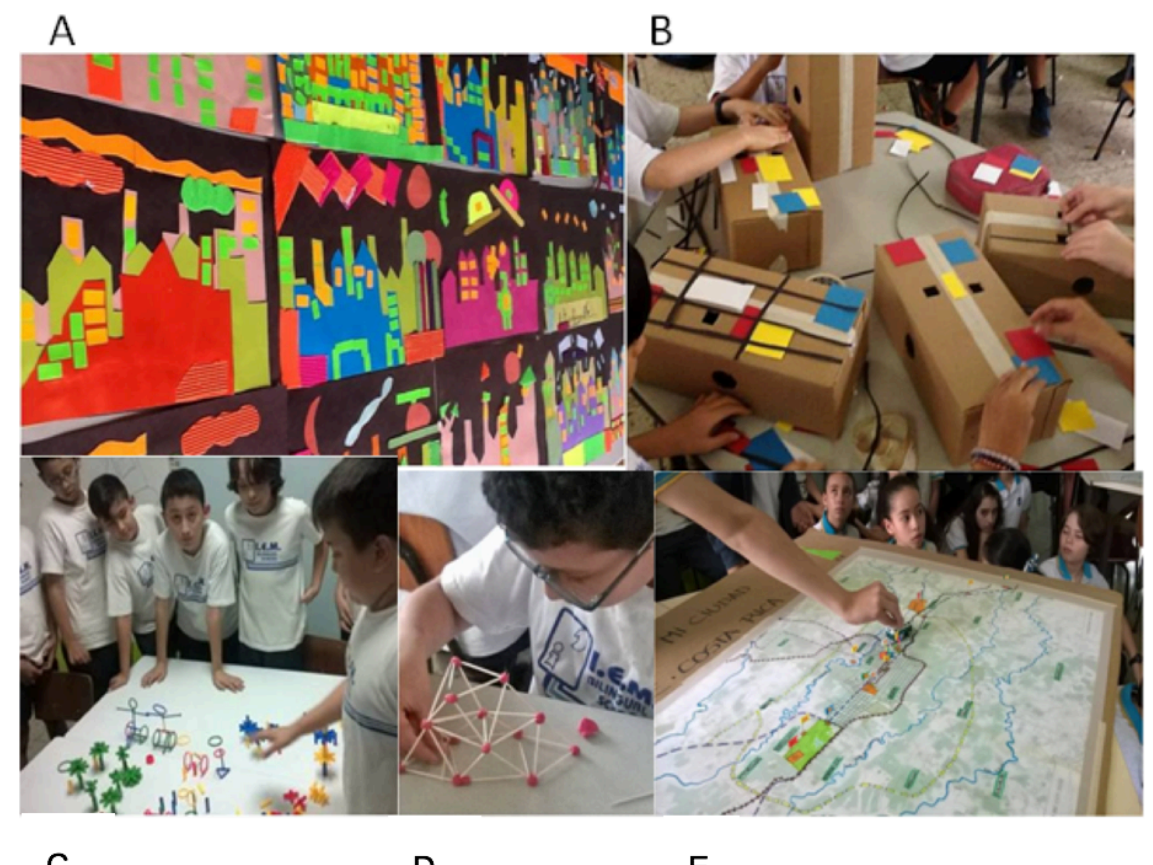

C

D

E

Figura 3. Taller Arqui-ticos del IEM Fuente: Elaboración propia.

El listado del temario a tratar en este último taller denominado Arqui-ticos se compone de varias etapas, este varía según el interés de la institución y de la realidad nacional por ejemplo el tema de los terremotos y de los volcanes que en nuestro país son una realidad permanente se realiza en apoyo a los planes de acción que se hacen al respecto. Los temas tratados en este taller aparecen en la tabla 2.

Adjunto link de Arqui-ticos en el anexo 1, donde se realiza entrevista al trabajo realizado. 
Tabla 2. Temas y subtemas tratados en el taller Arqui-ticos

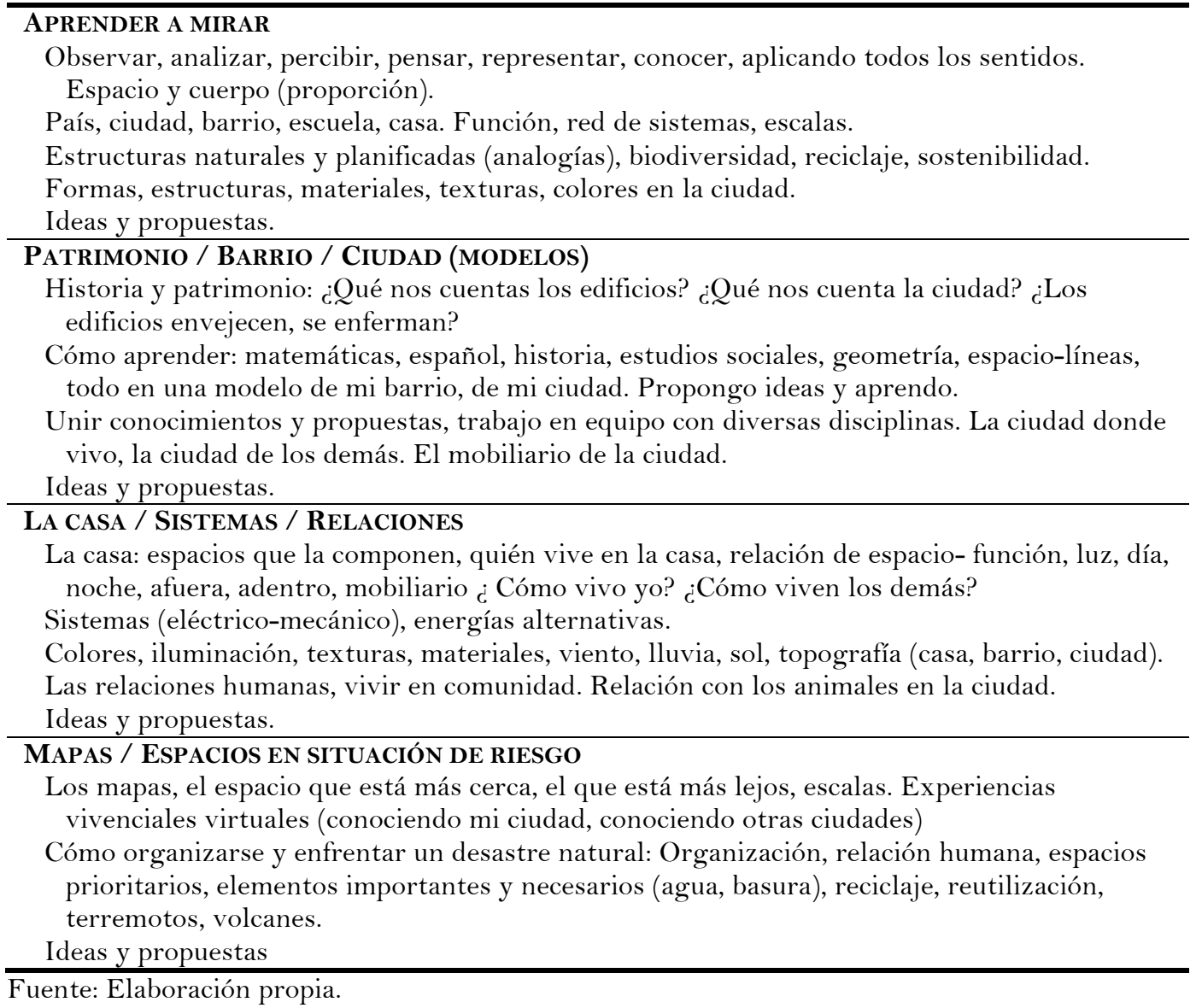

Fuente: Elaboración propia.

\section{Resultados}

Luego de realizar los talleres con el fin de ahondar en temas del entorno construido nacional, destacando en lo arquitectónico, la infraestructura, la ciudad y la vida en comunidad a través de talleres lúdico creativos y participativos, se puede indicar que los niños y jóvenes pudieron compartir intercambiando conocimientos e ideas en temas de ciudad como lo son: el transporte, cultura, movilidad, patrimonio, paisaje y accesibilidad, espacio público, entre otros. Temas que se plantearon con las siguientes preguntas realizadas a los participantes a través del desarrollo del guion de la obra de teatro y títeres denominada "Una comunidad para todos" y de la interacción de los personajes con el público: ¿Cómo le gustaría que fuera su barrio, escuela y/o comunidad?, ¿Le cambiarías algo? ¿Qué ideas tienes para tu ciudad? ¿Qué le falta a tu ciudad? ¿Cómo puedes ayudar a tu ciudad?

Se constató la gran participación activa de los participantes en cada ejemplo de taller y el sentido crítico y creativo a la hora de plantear soluciones y propuestas para la ciudad. También se pudo apreciar el disfrute de la experiencia y la alegría y entusiasmo de su participación. De esta manera se generaron propuestas e ideas urbanas de parte de los niños, niñas y jóvenes de las escuelas, de las comunidades, para mejorar el barrio, el entorno inmediato y la ciudad, así como entender la importancia y la responsabilidad como usuario de participar activamente de los cambios del barrio, de la comunidad, de la ciudad. 
En el Taller 1, realizado en 20 escuelas de atención prioritaria, se recopiló la información por parte de los realizadores profesionales con especialidad en los campos de la arquitectura, el psicodrama, el teatro, la educación y el diseño industrial en un tiempo de un mes para cada escuela. En el Taller 2, la información fue recopilada por los profesionales realizadores antes mencionados y por los estudiantes que apoyaron la propuesta, quienes realizaron su Trabajo Comunal Universitario (TCU) siendo asistentes de estos talleres, estudiantes de las carreras de Psicología, arquitectura, antropología, sociología y artes; esta recopilación se realizó durante los 10 días consecutivos que duró la actividad. En el Taller 3, la recopilación de ideas se realizó por parte de la facilitadora durante los cuatro meses que dura el curso, en forma paulatina conforme se fueron desarrollando las diversas temáticas del mismo. Esta información en todos los talleres se obtuvo en las siguientes modalidades: en dibujos, en forma escrita, construidas en modelos con material de reciclaje, en mapeo y participativas durante la presentación de teatro y títeres, y en forma oral para luego realizar un análisis de las propuestas en cada taller y agruparlas en tres grandes ejes temáticos: ambiente, socio-cultural y educativo. Se obtiene un mayor interés en propuestas ambientales lo cual coincide con la realidad de nuestro país Costa Rica, tema de mucho peso en la formación de los niños y jóvenes al tener un gran porcentaje del territorio declarados parques nacionales, lo que contrasta con la realidad al no estar reflejado este tema en la ciudad, específicamente en San José, el cual se evidencia en la opinión de los niños.

Algunas de las ideas y sugerencias obtenidas por los participantes son:

- Quiero una ciudad con colores.

- Quiero una ciudad para caminar con mi abuelo de la mano.

- Si el edificio me da para vivir, me debería dar para comer y hacerlo con el viento, el sol y el agua.

- Se necesitan espacios felices y divertidos.

- Lugares donde encontrar amigos.

- Un lugar donde compartir.

- La ciudad debe tener espacios para leer.

- Muchos lugares donde jugar y andar en bici.

- Sitios donde conversar.

- Un lugar para sonreír.

- Más lugares para hacer deportes.

- La ciudad debe prohibir menos y saludar más. Por ejemplo poniendo letreros que saluden a la gente cuando va a trabajar. En estos letreros además se podrían poner las poesías de los poetas del barrio y así conocerlos o también podrían tener los dibujos de muchos niños.

- Poder escuchar los pájaros, porque los buses hacen ruido.

- Decirle a las personas que no golpeen con las carteras a los niños cuando caminan en la ciudad. 
- Colocar más basureros, que funcione más seguido el tren y que usemos más la bicicleta.

- Se debe reciclar y reutilizar para ayudar al ambiente, pero tenemos que hacerlo todos a la vez.

- Una ciudad con árboles y muchas flores.

- Un parque donde vivan animales.

- Un parque con fuentes.

- Que los parques tengan agua y juegos para niños.

- Que la ciudad tenga más espacios para que la gente se reúna a conversar.

- Muchas ciclo vías para poder ir a la escuela.

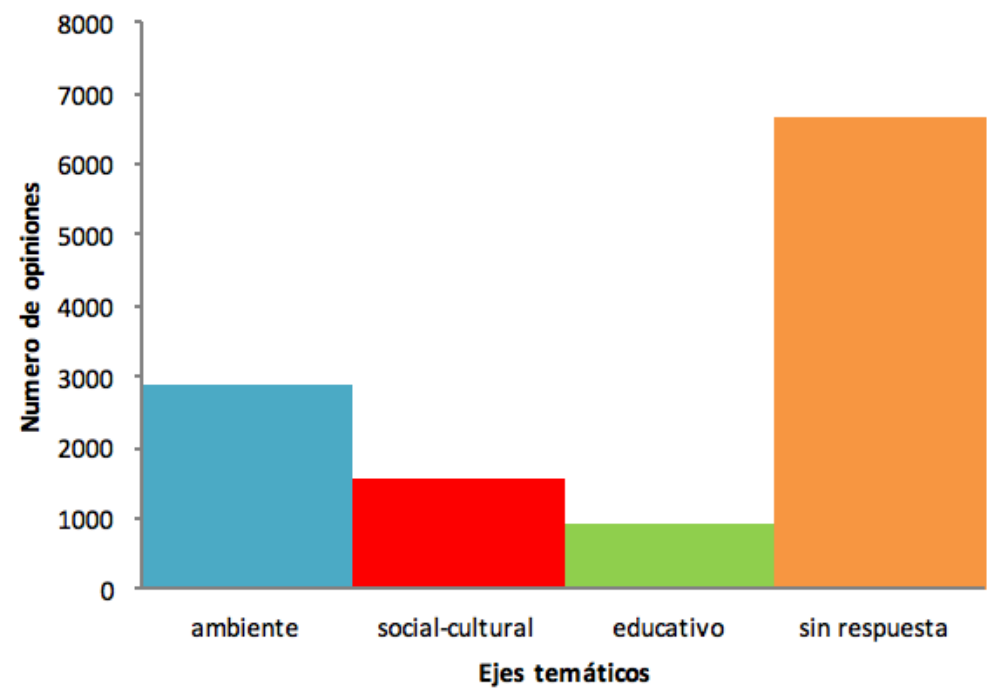

Gráfico 1. Opiniones de los participantes de los tres talleres según los ejes temáticos Fuente: Elaboración propia.

En el gráfico 1 se observan los resultados de opiniones y las temáticas abordadas de los participantes a los tres talleres: Taller 1, 20 Escuelas de Atención prioritaria con 643 estudiantes que dieron sus ideas para la ciudad en las temáticas de ambiente, sociocultural y educativo. Taller 2, "La ciudad a nuestra medida", tuvo una participación de 11400 personas (niños, jóvenes y adultos), de los cuales dieron respuesta a las temáticas de ambiente, socio-cultural y educativo 4700 estudiantes, 4300 no dieron su opinión por el factor tiempo de visita asignada y los restantes 2400 visitantes no dieron respuesta por ser público itinerante que participó solo observando. Taller 3, Arqui-ticos, 27 estudiantes quienes dieron su opinión en las temáticas de ambiente, socio-cultural y educativo.

Estos resultados reflejan el gran interés de esta población de participar y proponer acerca del tema ciudad, debemos propiciar más espacios para ello, para que esta participación sea más fuerte y permanente. Los gobiernos locales, escuelas, universidades deben incluir a los niños y jóvenes y hacerlos partícipes en todos sus programas a desarrollar. De igual manera acercar a profesionales y/o a agrupaciones de 
arquitectos que trabajen el tema de arquitectura y urbanismo con los niños para poder retroalimentar e intercambiar experiencias, planificar proyectos en conjunto y de esta manera unir esfuerzos logrando intercambio de opiniones entre los niños y jóvenes de la región. Dos ejemplos de acercamiento y de intercambio en este sentido son las agrupaciones ANDA (Arquitectura y Niñez de América) recién conformada desde el año 2016 que cuenta con 16 países de América Latina trabajando en conjunto, y la Red Ocara que opera desde Brasil, red latinoamericana de experiencias y proyectos sobre ciudad, arte, arquitectura y espacio público en los que participan niños y niñas.

Se sugiere documentar las opiniones obtenidas de los participantes en los talleres en cuanto a los temas de ciudad y espacio construido se refiere para utilizarlas en futuros diseños $\mathrm{y} / \mathrm{o}$ propuestas $\mathrm{y}$ facilitarlo a las entidades competentes, asimismo poder utilizarla en futuros proyectos.

De igual manera se sugiere promover relaciones y/o alianzas con diversas instituciones como lo pueden ser los Ministerios de Cultura, Ministerios de Educación, Municipalidades, Escuelas, Colegios y Universidades así como ONG's tanto a nivel nacional como internacional. Como ejemplo adjunto la opinión del área de la niñez de la Secretaría de Promoción Social de la Municipalidad de Rosario que señala en el artículo "La ciudad de los niños y las niñas", lo siguiente:

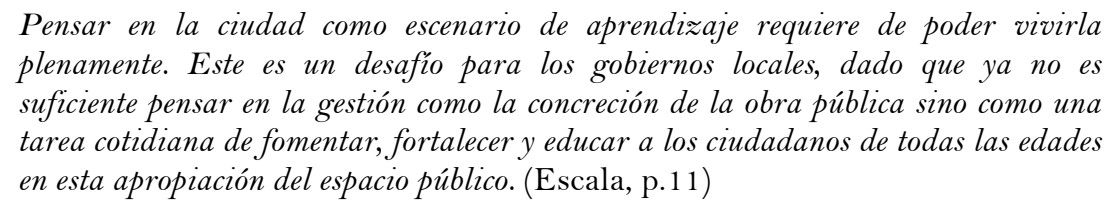

Dado lo anterior se entiende la importancia de generar redes de conocimiento, de trabajo y de intercambio en pro de la educación y formación de los niños, las niñas y la juventud del país como una necesidad urgente de transmitir conocimientos del espacio construido, de la arquitectura, del urbanismo y del medio ambiente. Esta población será el futuro usuario y responsable de la sostenibilidad de nuestras ciudades y de nuestro espacio construido próximo. Lo realizarán con conocimiento del tema y teniendo presente la siguiente ecuación: Educación + Juego + Compartir + Escuchar + Niños + Juventud + Aprendizaje + Crear + Conocimiento + Experiencia + Diversión $=$ Humanizar la ciudad. La arquitecta Joséphine Dusapin (2016) nos reafirma lo anteriormente comentado cuando indica: "Crear una conciencia hacia el futuro de nuestra sociedad es de suma importancia para desarrollar un sentimiento de pertenencia hacia el entorno que los rodea, un entendimiento para apropiárselo, valorarlo, respetarlo y poder decir y decidir su destino" (p. 57).

\section{Discusión y conclusión}

En los tres talleres descritos anteriormente, aunque fueron realizados en tiempos distintos, se refleja la necesidad y el interés en los niños, niñas y jóvenes de conocer, explorar y proponer en los temas de arquitectura y urbanismo, con el fin de ayudar a mejorar la ciudad incentivando la convivencia, apropiándose del espacio público, logrando ciudades más humanas, más cercanas con calidad de vida para todos. Facilitar a los niños y jóvenes un espacio para opinar y proponer al respecto es muy necesario y poco se hace. Realizar esta preparación desde los espacios que esta población utiliza diariamente como lo son las escuelas, el barrio, la comunidad, la casa, la familia, los 
amigos, permite compartir el parecer del arquitecto colombiano Antonio Manrique Gutiérrez (2007) cuando expresa lo siguiente en el artículo "Diseñando, arquitectura como educación":

\begin{abstract}
Si estamos de acuerdo en que el espacio es el gran protagonista de la arquitectura, entenderemos inmediatamente el valor que tiene para la vida de la sociedad el que los ciudadanos, desde niños, comprendan sensible y racionalmente, el sentido que da forma, lugar y tiempo a los espacios en que se habita. (p. 20)
\end{abstract}

Durante los diversos talleres se puede determinar cómo conciben la ciudad los niños y jóvenes, cuánto conocimiento poseen y si observan y caminan la ciudad o no. En la diversidad de la conformación de los grupos de niños de estos tres talleres que representan diferentes barrios, escuelas y comunidades, enriquece el trabajo grupal e individual, la gama de lo diverso, de lo diferente. Ellos expresan sus preocupaciones desde su perspectiva, desde su realidad, desde su conocimiento y experiencia, así se proponen ideas entre todos y/o individualmente.

En nuestra realidad son pocos los niños que experimentan la ciudad diariamente a pie, vivimos un mundo del bien llamado acelere, donde el transporte se realiza en medios cerrados (carros, buses, trenes), ya no existe la posibilidad de experimentar, de jugar y de caminar la ciudad. Por esto es importante abrir nuevas experiencias, nuevos sentidos (olfato, vista, tacto, gusto, sonidos) y de esta manera descubrir el entorno y la ciudad donde se habita, donde se vive sorprendiéndose seguramente de las cosas más sencillas y propias. Dusapin (2016) propone que:

\begin{abstract}
al ver la ciudad con otros ojos, caminar por ella, sus perspectivas se abren, se multiplican, para dejar campo a un mundo de múltiples posibles. Logran crear algo nuevo que no conocen pero que desean conocer y experimentar. Crean vida alrededor de este ideal de ciudad, incluyente, que promueve el caminar, los colores, la naturaleza, una experiencia cotidiana agradable. Se convierten en actores de este mundo, aprehendiendo su lugar de vida con una nueva perspectiva. (p. 59)
\end{abstract}

Al finalizar y comparar los resultados de los talleres realizados en los diferentes locaciones se concluye que los conocimientos adquiridos en los niños acerca de los temas propuestos: arquitectura, infraestructura y ciudad, se asimilaron generando opinión, sentido crítico y creativo en esta población así como entender que el trabajo en equipo es importante para las mejoras de la comunidad. Además se comprueba la alegría de ser partícipes en la construcción de propuestas urbanas. Definitivamente los aportes de este grupo deben ser escuchados y considerados, se deben hacer mayores esfuerzos por parte de los profesionales, organismos e instituciones para sensibilizar a los niños, niñas y jóvenes en los temas mencionados e integrarlos en propuestas específicas para que contribuyan con su opinión.

Se sugiere seguir realizando talleres en escuelas, colegios y comunidades del país. Talleres para preparar, compartir y recopilar ideas de los usuarios más pequeños de la ciudad, tarea que debe incorporarse fuertemente en nuestra región, con tanta desigualdad social y desintegración. Tarea que debe hacerse desde los gobiernos locales y diversos organismos involucrados de manera que se difunda fuertemente este proceso de sensibilización y educación en los niños y jóvenes. El grupo Somos Aldea de Chile expone en su página web (http://www.somosaldea.org/que-hacemos), disponible en los años 2015 y 2016, un objetivo común con lo que se presenta en este proyecto, el cual señala que el objetivo de estos talleres es crear instancias para aprender a observar el entorno críticamente, es decir, generar capacidades en las personas para pensar de 
manera informada los espacios que habitamos y activar el derecho a tener una voz sobre su diseño y construcción. Se constituyen como instancias de sensibilización, aprendizaje y empoderamiento para grupos que son o serán sujetos de proyectos de renovación urbana, de nueva infraestructura pública o como parte de una actividad formativa en el contexto escolar, universitario o comunitario. A través de actividades creativas de análisis del ambiente construido se alzan las aspiraciones sobre los lugares en que vivimos colaborando a generar más activistas del espacio.

Nos asombraríamos de las nuevas ideas que este grupo propone y que dejamos pasar los adultos, acciones que deben tratarse con seriedad. Para esto se realizan esfuerzos por parte de muchas agrupaciones de profesionales que luchan fuertemente tanto a nivel nacional como internacional en el campo de la educación de la Arquitectura para Niños con el fin de insertar este tema en el curriculum formal escolar, de intercambiar experiencias entre profesionales al respecto, lo cual ayudaría a hacer más cercanas y participativas las temáticas y llegaría a una mayor parte de la población de niños y de juventud, "La educación correcta consiste en despertar en el niño lo que hay en él, ayudarlo a desarrollarse y encaminar ese desarrollo en determinado sentido" (Vygotsky, 1999, p. 44)

Se debe educar para la construcción de espacios que permitan la vivencia humanizada en una ciudad, donde todos los temas mencionados inciden en una arquitectura comprometida con el desarrollo social, con la justicia social que se relaciona directamente cuando se respetan los derechos básicos de los niños y de las niñas, como lo indica la Convención sobre Los derechos del niño y la niña de las Naciones Unidas de 1989 entre muchos otros derechos, específicamente el respeto al derecho a ser oídos y a expresar su opinión, a ser integrados (Defensa de los Niños Internacional de Costa Rica, 1989). Es imperante y necesario incorporar a este grupo en las tomas decisiones urbanas desde tempranas edades haciéndoles partícipes en forma inclusiva porque todos somos parte de la ciudad, estas experiencias realizadas así lo reflejan y lo demandan, como también lo reafirma Francesco Tonucci, psicopedagogo más conocido como Frato en su obra "La ciudad de los niños, un nuevo modo de pensar la ciudad" de 2015:

\footnotetext{
...dar a los niños un papel de protagonistas, concederles la palabra, permitirles que expresen sus opiniones y colocarnos, nosotros los adultos, en la actitud de escuchar, de deseo de comprender y de voluntad de tener en cuenta lo que los niños dicen... Hacer hablar a los niños no significa pedirles que resuelvan los problemas de la ciudad. Significa, en cambio, aprender a tener en cuenta sus ideas y sus propuestas. (Tonucci, 2015, p. 173)
}

\section{Anexo 1}

Entrevista realizada a los niños inscritos en el curso de ARQUI-TICOS en el año 2016 por el periódico costarricense La Nación.

http://www.nacion.com/vivir/tendencias/Ninos-quieren-espacios-publicosdiversidad_O_1560044021.html

\section{Referencias}

Defensa de los Niños Internacional, sección Costa Rica, (1989). Convención sobre Los Derechos del Niño y la Niña. San José de Costa Rica: DNI COS. 
Dusapin, J. (2016). El aprendizaje de la mirada. Habitar, 88, 56-59.

Escala. (Eds.) (s.f.). La ciudad de los niños y las niñas. Recuperado de http:/ /www.revistaescala.com/index.php?option=com_content\&view=article\&id=2 13:1aciudad-de-los-ninos-y-las-ninas\&catid=60:educacion\&Itemid $=93$

Gutiérrez, A. M. (2007). Diseñando, arquitectura como educación. Escala: Arquitectura Latinoamericana, 214, 20-24.

Martín-Baró, I. (2004) Acción e ideología: Psicología social desde centroamérica. San Salvador: UCA editores.

Räsänen, J. (2014). Space, time, movement. The abc of architecture: Basics Concept, 2, 29-33.

Subirats, M. (2007). Ciudades educadoras: Un proyecto cargado de futuro. Participación Educativa, 6, $51-59$.

Tonucci, F. (2015). La ciudad de los niños, un nuevo modo de pensar la ciudad. Barcelona: GRAÓ.

Vygotsky, L. S. (1999). Imaginación y creación en la edad infantil. Buenos Aires: Nuestra América

\section{Breve CV de la autora}

\section{Ximena C. Pizarro}

Licenciada en Arquitectura, estudios en Universidad de Costa Rica y Universidad de las Ciencias y el Arte. Gestora y Productora de Actividades Culturales. Nace en Concepción Chile 1963 con nacionalidad actual costarricense. Ex miembro de la Junta Directiva del Colegio de Arquitectos de Costa Rica (Período 2008-2010 y 2014-2016) con el puesto de secretaría. Actualmente Coordinadora de la Comisión Espacio Construido y Niñez Costarricense del Colegio de Arquitectos de Costa Rica. Representante Oficial del Work Program Architecturte \& Children de la Unión Internacional de Arquitectos UIA. Gestora del Grupo A.N.D.A. Arquitectura y Niñez de América. Gestora de ARQUITICOS de Costa Rica. Presidenta de Grupo DIAR (Diseño Industrial y Arquitectura).Arquitecta por Costa Rica del Grupo Costa Rica Natural Design, empresa desarrolladora de proyectos de arquitectura (España - Costa Rica). Directora Ejecutiva Grupo Ticotíteres de Costa Rica agrupación cultural y artística dedicada a temas de la niñez y la juventud costarricense desde 1990. ORCID ID: 0000-0003-0031-9372. Email: xcpizarro@gmail.com. 